\begin{tabular}{ll}
\hline \hline MINING AND METALLURGY INSTITUTE BOR & ISSN: 2334-8836 \\
& UDK: 622 \\
\hline \hline
\end{tabular}

UDK: $621.967 .2: 622 \cdot 271(045)=20$

DOI:10.5937/MMEB1302053R

Branislav Rajković, Zoran Ilić, Radomir Mijovic ${ }^{*}$

\title{
TRANSIENT TIME DETERMINATION AT START UP OF BELT CONVEYOR FOR ORE T.109
}

\begin{abstract}
This work gives the method of transient time determination of the belt conveyor in the case of its start up in the example of a belt conveyor for ore at the open pit "Veliki Krivelj" with capacity of $2000 \mathrm{t} / \mathrm{h}$.

The analysis was made by calculation procedure and it represents a universal calculation method of conveyor belts whose results are indispensable for verification the reliable operation of selected electric motors during the start up of belt conveyor.

Also, technical characteristics of all components of drive units are given as well as their layout.

Keywords: belt conveyor for ore, transient time, drive unit
\end{abstract}

\section{INTRODUCTION}

Asynchronous short-circuited electric motors, i.e. asynchronous electric motors with wound rotor are most commonly used as a belt conveyor drive. During the start up of a belt conveyor the start current may be several times greater than rated current. Therefore it is desirable that the transient time at the start up of a belt conveyor is as short as possible, i.e. the electric motor reaches its rated speed as soon as possible. If the transient time is too long, the electric motor will fail to start due to activation of protection devices (if properly adjusted), otherwise electric motor will burn out.

The belt conveyor for ore T.109 was designed for transportation the primary crushed ore at the open pit "Veliki Krivelj" Bor from the primary crusher (pos. T.102.100.2) to the open storage for ore [1]. There are two identical drive units predicted for the belt conveyor drive, each driving one drive pulley. Selection of drive units is the result of calculation procedure which is not considered in this paperwork, but certain analyses of belt conveyor T.109 regarding to this matter are given in [2]. However, the results of these previous calculations are input data for determination of transient time during belt conveyor start up. It should be mentioned that a complete calculation of belt conveyor generally contains calculation of different load cases, as well as dynamic operation regimes like start up, stopping and backstopping [3]. The results obtained by analytical procedures, given in the above mentioned paper, may be useful in selection of computer software for belt conveyor calculation which application is undoubtedly the future in this field.

Drive units of belt conveyor T.109 are placed at the return side of belt conveyor in the scope of drive-take up-discharge station at discharge end of belt conveyor.

The layout of the belt conveyor drive units is given in Figure 1.

\section{TECHNICAL DESCRIPTION OF BELT CONVEYOR DRIVE UNITS}

The belt conveyor drive unit consists of an electric motor, a coupling between the electric motor and gear, the gear and coupling between gear and drive pulley.

* Mining and Metallurgy Institute Bor 
Technical characteristics of electric motor:

- Manufacturer: Sever Subotica

- Label: ZPN 6170

- Rated power: $P=450[\mathrm{~kW}]$

- Speed: $n=982\left[\mathrm{~min}^{-1}\right]$

- Power factor: $\cos \varphi=0,9[-]$

- Rated current: $I_{n}=51,5[\mathrm{~A}]$

- Rated torque: $M_{n}=4376[\mathrm{Nm}]$

- Start current and rated current ratio:

$$
I_{p} / I_{n}=2[-]
$$

- Maximum torque and rated torque $\mathrm{r}$ atio: $M_{\max } / M_{n}=2[-]$

- Mass: $m=4200[\mathrm{~kg}]$

Technical characteristics of coupling between electric motor and gear:

- Manufacturer: Falk

- Label: 1110T10

- Rated torque: $M_{n}=9320[\mathrm{Nm}]$

- Maximum speed: $n_{\max }=2250[\mathrm{rpm}]$
Technical characteristics of gear:

- Manufacturer: Falk

- Label: 485-A2

- Rated power: $P=788[\mathrm{~kW}]$

- Ratio: $i=15.44[-]$

- Mass: $m_{\text {red }}=2857.6[\mathrm{~kg}]$

Technical characteristics of coupling between gear and drive unit:

- Manufacturer: Falk

- Label: 1180T10

- Rated torque: $M_{n}=103000[\mathrm{Nm}]$

- Maximum speed: $n_{\max }=1100[\mathrm{rpm}]$

The belt conveyor T.109 is driven by two identical drive units containing specified components. At both drive units an asynchronous slip ring electric motor drives gear via an elastic coupling, and gear drives the drive pulley via another elastic coupling moving the belt of belt conveyor itself. So, during the operation of belt conveyor, a permanent mechanical connection between electric motor and drive pulley is realized.

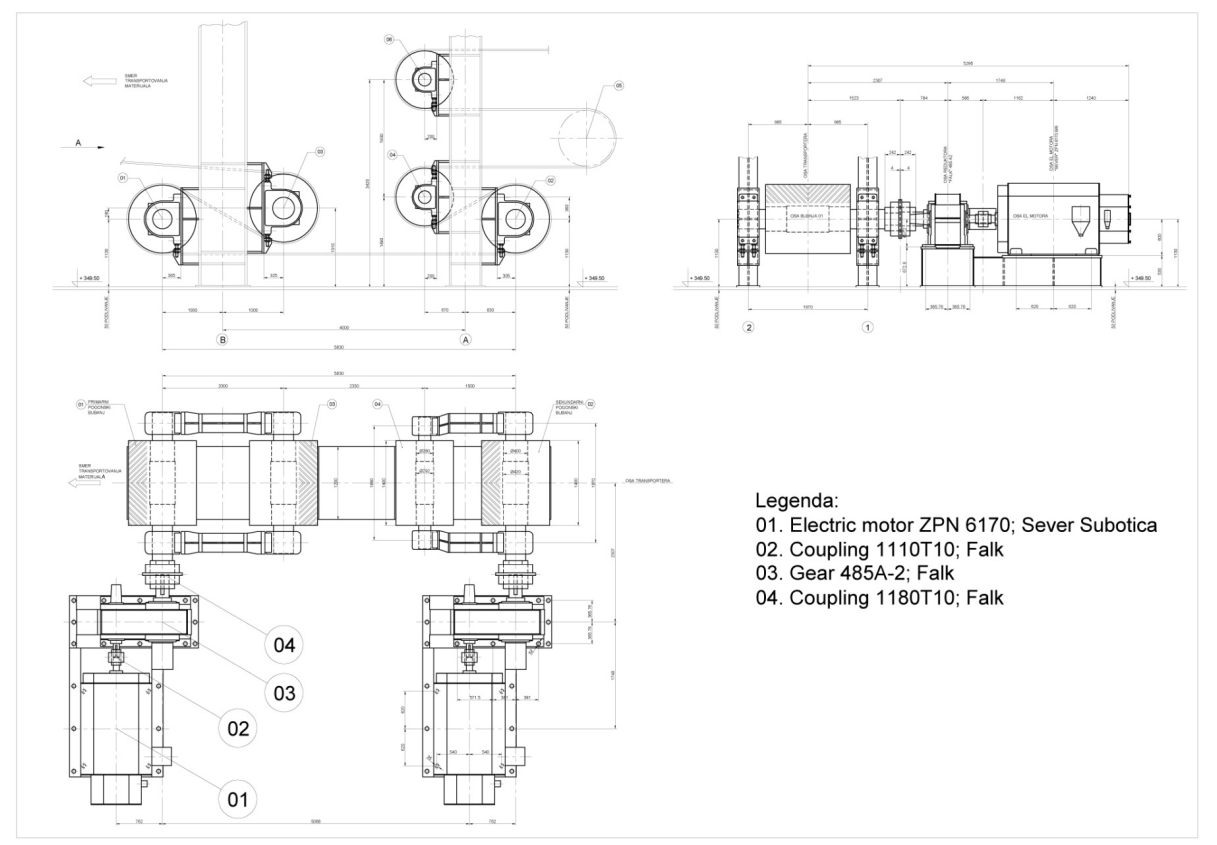

Figure 1 Drive units of belt conveyor T.109 


\section{TRANSIENT TIME CALCULATION AT START UP OF BELT CONVEYOR T.109}

1. Transient time calculation is aimed for determination the transient time at start up of loaded belt conveyor according to [4].

2. Transient time at start up of belt conveyor

$$
t_{p o l}=\frac{I_{s v} \cdot w_{E M}}{M_{s r, p}-M_{s t, p}}[s]=3,4[s]
$$

3. Inertia moment of belt conveyor movable masses reduced to the electric motor shaft

$$
\begin{aligned}
I_{s v} & =\delta_{r o t} \cdot\left(I_{r}+I_{s p}\right)+ \\
& +\frac{m_{p, t r} \cdot r^{2}}{i_{s, r e d}^{2} \cdot \eta_{p}}\left[\mathrm{kgm}^{2}\right]= \\
& =112.3\left[\mathrm{kgm}^{2}\right]
\end{aligned}
$$

where:

$$
\begin{aligned}
& \delta_{\text {rot }}=1.15 \text { - coefficient } \\
& I_{r}=41\left[\mathrm{kgm}^{2}\right] \text { - inertia moment of } \\
& m_{p, t r}=51035.35[\mathrm{~kg}]-\text { reduced }
\end{aligned}
$$

4. Efficiency during start up of electric motor

$$
\eta_{p}=1-(1-\eta) k_{p . p} \cdot c_{s m}=0.865
$$

where:

$$
\begin{aligned}
& \eta=0.85-\text { efficiency of drive unit } \\
& k_{p . p}=1.5-\text { coefficient of static resista- } \\
& \text { nce increase during start up } \\
& c_{s m}=0.6 \begin{array}{l}
\text { - coefficient of possible resis- } \\
\text { tance reduction at belt mo- } \\
\text { tion }
\end{array}
\end{aligned}
$$

5. Angular speed of electric motor

$$
w_{E M}=\frac{n_{E M} \cdot \pi}{30}=102.8\left[\frac{\mathrm{rad}}{\mathrm{s}}\right]
$$

where:

$$
n_{E M}=982\left[\mathrm{~min}^{-1}\right]-\text { electric motor }
$$

6. Average torque of electric motor during the start up

$$
\begin{aligned}
M_{s r, p} & =\frac{M_{p . \max }+M_{p . \min }}{2}= \\
& =\frac{2 M_{n}+1.1 M_{n}}{2}=1.55 \cdot M_{n}= \\
& =6782.8[\mathrm{Nm}]
\end{aligned}
$$

where:

$$
\begin{gathered}
M_{n}=4376[\mathrm{Nm}] \text { - electric motor rated } \\
\text { torque }
\end{gathered}
$$

7. Static torque during start reduced to the electric motor shaft

where:

$$
M_{\text {st }, p}=\frac{W \cdot r}{i_{\text {s.red }} \cdot \eta_{p}}=3383.5[\mathrm{Nm}]
$$

$W=87115,5[N]$ - static resistance force due to pulling force during start up of belt conveyor

\section{DISCUSSION OF CALCULATION}

Selection of drive unit elements for belt conveyor drive is done according to the static resistance force due to the pulling force for nominal operation conditions. However, electric motor verification in the period of start up is done according to the static resistance force due to the pulling force during the start up of belt conveyor regarding the increased resistance at belt conveyor start. Also, it is assumed that the belt conveyor starts loaded by material. In such a way, electric motor transient time determination is done for the most unfavourable working conditions. 
As it is apparent from electric motor technical characteristics, the start current and rated current ratio is $2: 1$ meaning that the electric motor heating is significantly higher during the start than it is in nominal operation regime. During the start the electric motor has to overcome, beside the resistance forces of belt motion, the inertial forces due to the start up of belt, material on the belt conveyor and rotating masses of carrying and returning rolls as well as rotating masses of all pulleys. Nevertheless, the transient time of electric motor start up has to be such that its burning does not happen.

\section{CONCLUSION}

Transient time of the electric motor start up, obtained by calculation, amounts $3.4 \mathrm{sec}$ and it is within recommended limits for transient time of belt conveyor start up, which in this case amounts 3 to $6 \mathrm{sec}$, so it can be concluded that the electric motors were designed correctly.

Calculation procedure shown here refers to the drive units with mechanical couplings between electric motors and drive pulleys. Beside mechanical couplings, hydraulic couplings are also used for belt conveyor drive as a protection from electric motor overload.

\section{REFERENCES}

[1] "Detail Mechanical Design of Transportation System for Ore from Transfer Station to the Conveyor at the Location of Open Storage for Ore"; Mining and Metallurgy Institute Bor; Bureau MEGA; 2011. (in Serbian)

[2] B. Rajković, Z. Ilić, R. Mijović: „Power and Load Distribution of Belt Conveyor for Ore with Two Driving Pulleys"; $43^{\text {rd }}$ International October Conference on Mining and Metallurgy; 12-15 October 2011; Kladovo, Serbia

[3] B. Rajković, Z. Ilić, R. Mijović: „Phenomenon of Reversal Motion of Belt Conveyor for Ore T.109 and Prevention of This Phenomenon"; Mining Engineering, 1(2012); Mining and Metallurgy Institute Bor

[4] S. Tošić: Calculation of Continuous Transportation Appliances and Lifting Devices, Mechanical Faculty Belgrade, 1994. (in Serbian) 


\begin{tabular}{ll}
\hline \hline INSTITUT ZA RUDARSTVO I METALURGIJU BOR & ISSN: 2334-8836 \\
& UDK: 622 \\
\hline \hline
\end{tabular}

\section{ODREĐIVANJE VREMENA TRAJANJA PRELAZNOG REŽIMA PRI STARTOVANJU TRAKASTOG TRANSPORTERA ZA RUDU T.109}

Izvod

U ovom radu je, na primeru trakastog transportera za rudu na površinskom kopu "Veliki Krivelj” sa kapacitetom $2000 \mathrm{t} / \mathrm{h}$, data metoda određivanja vremena trajanja prelaznog režima trakastog transportera u slučaju njegovog startovanja.

Analiza je urađena računskim putem i predstavlja univerzalni metod proračuna trakastih transportera čiji su rezultati neophodni za proveru pouzdanog rada izabranih elektromotora pri startovanju transportera.

Takođe su date tehničke karakteristike svih elemenata pogonskih grupa i njihov graficki prikaz.

Ključne reči: trakasti transporter za rudu, vreme prelaznog režima, pogonska grupa

\section{UVOD}

Za pokretanje trakastih transportera najčešće se koriste asinhroni kratkospojeni elektromotori odnosno asinhroni elektromotori sa namotanim rotorom. Prilikom startovanja elektromotora polazna struja može biti i nekoliko puta veća od struje u nominalnom režimu rada. Zbog toga je poželjno da vreme startovanja elektromotora bude što kraće odnosno da on postigne svoju nominalnu brzinu što pre. Ukoliko je vreme startovanja predugo elektromotor neće uspeti da startuje (pri ispravnom podešavanju), odnosno elektromotora će pregoreti.

Trakasti transporter za rudu T.109 projektovan je za transport primarno izdrobljene rude na površinskom kopu "Veliki Krivelj” Bor od drobilice (poz. T.102.100.2) do otvorenog sklada za rudu [1]. Za pokretanje trake transportera su predviđene dve identične pogonske grupe od kojih svaka pokreće po jedan pogonski bubanj. Izbor pogonskih grupa je rezultat proračunskog postupka koji niji razmatran $\mathrm{u}$ ovom radu ali određene analize trakastog transportera T.109 koje se odnose na ovu materiju su date u [2]. Međutim, rezultati ovih prethodnih proračuna su ulazni podaci za određivanje vremena trajanja prelaznog režima pri startovanju trakastog transportera. Treba napomenuti da kompletan proračun jednog trakastog transportera uopšte uzev sadrži proračun različitih slučajeva opterećenja, kao i dinamičke radne režime kao što su startovanje, zaustavljanje i zadržavanje [3]. Rezultati dobijeni analitičkim postupcima datim u napred navedenim radovima mogu biti korisni pri izboru kompjuterskih programa za proračun trakastih transportera čija primena nesumnjivo predstavlja budućnost na ovom polju.

Pogonske grupe smeštene su na povratnoj strani transportera u okviru pogonskozatezno-istovarne stanice na istovarnom kraju transportera.

Prikaz pogonskih grupa trakastog transportera dat je na crtežu 1 .

\footnotetext{
* Institut za rudarstvo i metalurgiju Bor
} 


\section{TEHNIČKI OPIS POGONSKIH \\ GRUPA TRAKASTOG TRANSPORTERA}

Pogonska grupa trakastog transportera sastoji se od elektromotora, spojnice između elektromotora i reduktora, reduktora i spojnice između reduktora i pogonskog bubnja.

Tehničke karakteristike elektromotora:

- Proizvođač: Sever Subotica

- Oznaka: ZPN 6170

- Snaga: $P=450[k W]$

- Broj obrtaja: $n=982\left[\mathrm{~min}^{-1}\right]$

- Faktor snage: $\cos \varphi=0,9[-]$

- Nominalna struja: $I_{n}=51,5[\mathrm{~A}]$

- Nominalni moment: $M_{n}=4376[\mathrm{Nm}]$

- Odnos polazne i nominalne struje: $I_{p} / I_{n}=2[-]$

- Odnos maksimalnog i nominalnog momenta: $M_{\max } / M_{n}=2[-]$

- Masa $: m=4200[\mathrm{~kg}]$

Tehničke karakteristike spojnice između elektromotora i reduktora:

- Proizvođač: Falk

- Oznaka: 1110T10

- Nominalni moment: $M_{n}=9320[\mathrm{Nm}]$

- Maksimalna brzina:

$n_{\max }=2250[\mathrm{rpm}]$
Tehničke karakteristike reduktora:

- Proizvođač: Falk

- Oznaka: 485-A2

- Snaga: $P=788[k W]$

- Prenosni odnos: $i=15,44[-]$

- Masa: $m_{\text {red }}=2857,6[\mathrm{~kg}]$

Tehničke karakteristike spojnice između reduktora i pogonskog bubnja:

- Proizvođač: Falk

- Oznaka: 1180T10

- Nominalni moment:

$M_{n}=103000[\mathrm{Nm}]$

- Maksimalna brzina:

$n_{\text {max }}=1100[\mathrm{rpm}]$

Trakasti transporter T 109 pokreću dve identične pogonske grupe koje sadrže navedene komponente. $U$ obe pogonske grupe asinhroni kliznokolutni elektromotor pogoni preko elastične spojnice reduktor, a ovaj preko druge elastične spojnice pogoni pogonski bubanj preko koga se pokreće traka samog trakastog transportera. Dakle tokom rada trakastog transportera ostvaruje se neprekidna mehanička veza između elektromotora i pogonskog bubnja

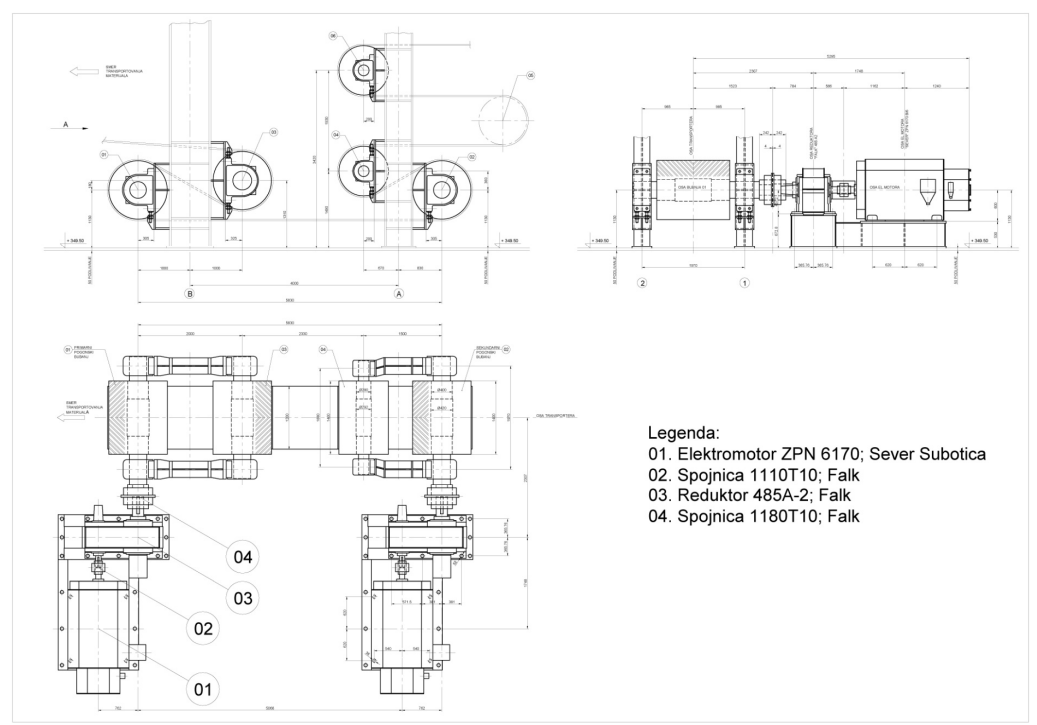

Crtež 1. Pogonske grupe trakastog transportera T.109 


\section{PRORAČUN VREMENA STARTO- VANJA TRANSPORTERA T.109}

1. Proračun vremena startovanja ima za cilj određivanje trajanja prelaznog režima transportera opterećenog materijalom pri startovanju i dat je prema [4].

2. Vreme puštanja transportera u pogon

$$
t_{p o l}=\frac{I_{s v} \cdot w_{E M}}{M_{s r, p}-M_{s t, p}}[s]=3,4[s]
$$

3. Moment inercije pokretnih masa transportera svedenih na vratilo elektromotora

$$
\begin{aligned}
I_{s v} & =\delta_{\text {rot }} \cdot\left(I_{r}+I_{s p}\right)+ \\
& +\frac{m_{p, t r} \cdot r^{2}}{i_{s, r e d}^{2} \cdot \eta_{p}}\left[\mathrm{kgm}^{2}\right]= \\
& =112,3\left[\mathrm{kgm}^{2}\right]
\end{aligned}
$$

gde su:

$$
\begin{gathered}
\begin{array}{c}
\delta_{\text {rot }}=1,15-\text { koeficijent } \\
I_{r}=41\left[\mathrm{kgm}^{2}\right]-\text { moment inercije ro- } \\
\text { tora elektromotora }
\end{array} \\
\begin{array}{c}
I_{s p}=0,270\left[\mathrm{kgm}^{2}\right] \\
\text {-moment inercije } \\
\text { spojnice između } \\
\text { elektromotora i } \\
\text { reduktora }
\end{array} \\
\begin{array}{c}
\text { pokretnih delova transportera po } \\
\text { bubnju } \quad \text { bubnja }
\end{array} \\
\begin{array}{c}
r=0,512[\mathrm{~m}] \text {-poluprečnik pogonskog } \\
i_{\text {s.red }}=15,44 \text {-prenosni odnos reduk- } \\
\text { tora }
\end{array} \\
\begin{array}{c}
\eta_{p}=0,865-\text { stepen korisnosti pri } \\
\text { puštanju }
\end{array}
\end{gathered}
$$

4. Stepen korisnog dejstva pogona u period puštanja elektromotora pri puštanju

$$
\eta_{p}=1-(1-\eta) k_{p . p} \cdot c_{s m}=0,865
$$

gde su:

$$
\begin{gathered}
\eta=0,85 \text { - stepen korisnog dejstva po- } \\
\text { gona } \\
k_{p . p}=1,5 \text { - koeficijent povećanja } \\
\text { statičkih otpora pri puštanju }
\end{gathered}
$$

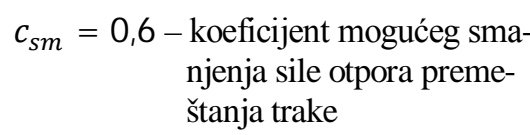

5. Ugaona brzina elektromotora

$$
w_{E M}=\frac{n_{E M} \cdot \pi}{30}=102,8\left[\frac{\mathrm{rad}}{\mathrm{s}}\right]
$$

gde je:

$$
\begin{gathered}
n_{E M}=982\left[\mathrm{~min}^{-1}\right]-\text { broj obrtaja elek- } \\
\text { tromotora }
\end{gathered}
$$

6. Srednji moment puštanja elektromotora $\mathrm{u}$ pogon

$$
\begin{aligned}
M_{s r, p} & =\frac{M_{p . \max }+M_{p . \min }}{2} \\
& =\frac{2 M_{n}+1,1 M_{n}}{2}=1,55 \cdot M_{n} \\
& =6782,8[\mathrm{Nm}]
\end{aligned}
$$

gde je:

$$
M_{n}=4376[\mathrm{Nm}]-\begin{gathered}
\text {-nominalni moment } \\
\text { elektromotora }
\end{gathered}
$$

7. Statički moment pri puštanju u pogon sveden na vratilo elektromotora

$$
M_{s t, p}=\frac{W \cdot r}{i_{\text {s.red }} \cdot \eta_{p}}=3383,5[\mathrm{Nm}]
$$

gde je:

$$
\begin{aligned}
W=87115,5[N]- & \text { sila statičkog opte- } \\
& \text { rećenja usled sile } \\
& \text { vuče pri puštanju } \\
& \text { transportera u po- } \\
& \text { gon }
\end{aligned}
$$

\section{DISKUSIJA PRORAČUNA}

Izbor pogonskih elemenata za pogon trake trakastog transportera vrši se prema sili statičkog opterećenja usled sile vuče za nominalne radne uslove. Međutim, provera izbora elektromotora $\mathrm{u}$ periodu puštanja $\mathrm{u}$ rad vrši se prema sili statičkog opterećenja usled sile vuče pri puštanju transportera u 
pogon koja se određuje uzimajući u obzir povećane otpore pri startu transportera. Takođe, pretpostavlja se da se startovanje transportera odvija sa transporterom koji je pun sa materijalom. Na taj način određivanje vremena startovanja elektromotora vrši se za najnepovoljnije radne uslove.

Kao što se iz podataka o tehničkim karakteristikama elektromotora vidi odnos polazne i nominalne struje je $2: 1$ što znači da je znatno intenzivnije zagrevanje elektromotora pri startovanju od zagrevanja elektromotora u nominalnom radnom režimu. Prilikom startovanja elektromotora on mora da savlada osim otpora kretanju trake transportera i silu inercije od pokretanja trake, materijala na transporteru i obrtnih masa nosećih i povratnih rolni kao i obrtnih masa svih bubnjeva. Pri tome vreme trajanja startovanja elektromotora mora biti takvo da ne dođe do njegovog pregorevanja.

\section{ZAKLJUČAK}

Proračunom dobijeno vreme startovanja elektromotora od 3,4 sec nalazi se u preporučenim granicama za vreme startovanja koje u ovom slučaju iznosi od 3 do 6 sec, tako da se može zaključiti da su elektromotori ispravno dimenzionisani.

Prikazana proračunska procedura odnosi se na pogon transportera sa mehaničkim spojnicama između elektromotora i pogonskog bubnja. Osim mehaničkih spojnica za pogon trakastih transportera primenjuju se $\mathrm{i}$ hidrauličke spojnice u svrhu zaštite elektromotora pri startovanju od preopterećenja.

\section{LITERATURA}

[1] „Tehnički mašinski projekat transportnog sistema za rudu od presipne stanice do transportera na lokaciji otvorenog sklada za rudu"; Institut za rudarstvo i metalurgiju Bor; Biro MEGA; 2011.

[2] B. Rajković, Z. Ilić, R. Mijović: „Power and Load Distribution of Belt Conveyor for Ore with Two Driving Pulleys"; $43^{\text {rd International October }}$ Conference on Mining and Metallurgy; 12-15 October 2011; Kladovo, Serbia

[3] B. Rajković, Z. Ilić, R. Mijović, „Pojava suprotnosmernog kretanja trakastog transportera za rudu T.109 i sprečavanje ove pojave"; Časopis Rudarski radovi 1(2012); Institut za rudarstvo i metalurgiju Bor; Bor

[4] S. Tošić, Proračun mašina neprekidnog transporta i dizaličnih uređaja, Mašinski Fakultet Beograd, 1994. 